\title{
Coenzyme Q10 and Vitamin D Interventions Could Ameliorate COVID-19 Related Cellular Bioenergetic Dysfunction and Cytokine Storms
}

\author{
Darshna Yagnik* \\ Middlesex University, Department of Natural Sciences, School of Science and Technology, The Burroughs, London, NW4 4BT, UK
}

Article Info

\section{Article Notes}

Received: July 05, 2021

Accepted: July 30, 2021

\section{${ }^{*}$ Correspondence:}

*Dr. Darshna Yagnik, Middlesex University, Department of Natural Sciences, School of Science and Technology, The Burroughs, London, NW4 4BT, UK; Email: d.yagnik@mdx. ac.uk.

C 2021 Yagnik D. This article is distributed under the terms of the Creative Commons Attribution 4.0 International License.

\section{Keywords}

Vitamin D

Coenzyme Q10

COVID-19

SARS-CoV-2

Mitochondria

Inflammation

Cytokine storms

Monocytes

\section{ABSTRACT}

The immune response to SARS-CoV-2 varies from asymptomatic or mild symptoms of high temperature, muscle aches and coughs lasting 7 to 14 days to lower respiratory tract infections leading to pneumonia and serious respiratory distress as well as long COVID-19. Complications occur due to an abnormal immune response which involves upregulation of multiple cytokines leading to sustained inflammation which results in the spread of infection to vital organs. The double vaccine roll out has been rapid however vaccine mediated antibodies are not $100 \%$ effective against future coronavirus variants which may become increasingly more resistant and easily transmissible to overcome host immunity. Invariably supportive therapies will be needed. Research has shown that coenzyme Q10 and vitamin $D$ deficiencies can have detrimental effects on immune cell defence, function and cytokine secretion promoting inflammation and sepsis especially against microbes. Early interventions including supplementation of these factors could mitigate cellular dysfunction especially in relation to mitochondria bioenergetics and help maintain cell immunity. This is particularly important as chronically ill COVID-19 patients seem to display abnormal immune cell phenotypes in infected organs indicating this could contribute to disease progression. The immune response and proposed roles of Vitamin $D$ and Coenzyme Q10 in COVID-19 are discussed.

\section{Introduction}

A novel highly contagious severe acute respiratory syndrome coronavirus 2 (SARS-CoV-2) which causes coronavirus disease (COVID-19) first emerged in late 2019 allegedly from a seafood market located in the Wuhan province of China. The virus has rapidly spread all over the world causing a historical pandemic resulting in nearly 4 million deaths and 181 million infections worldwide ${ }^{1}$. SARS-CoV-2 is proving a struggle to control as it is air borne, easily transmitted and has continually mutated into more virulent versions since the pandemic first began. Also, the human immune response to the virus is extremely varied ranging from asymptomatic, mild flu like symptoms through to cytokine storms and life-threatening Acute Respiratory Distress Syndrome (ARDS) 2,3.

COVID-19 symptoms include fever, high temperature, cough, lethargy and acute respiratory distress. The immune cells within the blood can also be affected by COVID-19 leading to lymphopenia, leukopenia, thrombopenia and anemia. In severe disease, immunologically there is an increase in systemic inflammation caused by the release of immune cell derived cytokines such as CRP, IL-6, TNF $\alpha$ and chemokines CCL2, CCL3, CXCL10 $0^{4-7}$. In addition 
patients with underlying co-morbidities such as diabetes, cardiovascular disease, asthma, renal or pulmonary disease could develop severe complications including sepsis and multi organ failure ${ }^{8}$. The risk of mortality from COVID-19 in patients increases with age, male sex, obesity, diabetes, hypertension and those originating from black, Asian or minority ethnic (BAME) backgrounds ${ }^{9,10}$. Emergency vaccines using new mRNA technology or adenoviruses which are mostly gene based have been rapidly rolled out across the world, their effectiveness will be tested in the days to come. To note there is some seasonal variability in SARS-CoV-2 infections and relative transmission has been linked to seasonal humidity levels which could mask the measure of vaccine effectiveness particularly in the summer seasons $\mathrm{s}^{11}$. Despite the vaccine roll out it seems inevitable that new more potent mutant strains of the virus will evolve in the near future, continue to plague the population and evade human immunity. Hence any early interventions that could potentially promote patient recovery from COVID-19 in its numerous clinical manifestations, are of particular interest.

\section{ACE2 Hijack by SARS-CoV-2}

The receptor for SARS-CoV-2 on human cells is angiotensin-converting enzyme 2 (ACE2) which is the binding site for the viral spike glycoprotein that allows the virus to bind to cells located in the nose and mouth during infection. Once the virus engages with the ACE2 receptors, attachment occurs followed by virus internalization and replication. The conversion of angiotensin II to angiotensin 1-7 is catalysed by ACE2. This leads to regulation of cardiac and kidney function via the renin angiotensin system (RAC) by modulating specific vasoconstrictors and dilators ${ }^{12}$. High ACE2 expression is detected on lung epithelial cells compared with low levels on vascular endothelial cells of the lungs and kidneys. ACE2 expression on blood cells is low except for type 1 activated monocytes but higher on liver kupffer cells and lung alveolar macrophages. High SARS-CoV-2 receptor expression on these cells could explain why lung and liver involvement occurs and perhaps why alveolar macrophages display abnormal phenotypes during severe COVID-19 pneumonia ${ }^{13}$. Since ACE2 is the receptor for SARS-CoV-2, one may hypothesise that lower expression would be ideal and could help combat infections. However high expression of ACE2 is actually beneficial to humans as it controls kidney and heart function. Membrane ACE2 is anti-inflammatory as shown in mice models and following SARS-CoV2 infection ACE2 is downregulated in lungs which could account for the rapid kidney, heart and lung damage observed in severe COVD-19 ${ }^{14}$. There is also growing evidence that COVID-19 has severe vascular manifestations as well as endothelitis with impaired microcirculatory function.

\section{Mitochondrial Hijack by SARS-CoV-2}

The human body consists of trillions of cells which have specific functions. Most eukaryotic cells contain mitochondria which function as the cell energy producing centres. They regulate carbohydrate, amino acid and fatty acid metabolism ${ }^{15}$. Mitochondria are modulated by internal cell dynamics which can be affected by metabolic diseases, cancer and viral infections. Viral proteins use mitochondria to survive and replicate. Indeed, cell release of antiviral interferon requires activation of the mitochondria's specific antiviral signalling proteins through mechanisms that involve $\mathrm{NFkB}^{16}$.

Viruses are by nature, highly manipulative of cells and can hijack mitochondrial structures impacting their cellular functions. Strikingly viruses including SARS-CoV-2 can change mitochondria membrane potential, ion permeability, calcium levels, cause induction of oxygen species and cell oxidative stress. In order to survive once within infected cells, viruses continue to alter mitochondrial DNA, also its distribution and disrupt the cell's overall antiviral capacity by switching the cytokine response to inflammatory rather than anti-viral ${ }^{17-20}$.

The Role of Coenzyme Q10 in Mitochondria Bioenergetics During Viral Infections

Coenzyme Q10 (CoQ10) is a redox quinoid component of the mitochondria electron transport chain and is found in all respiring eukaryotic cells. Its function is to generate cell energy (ATP) through aerobic respiration ${ }^{21}$. The levels of CoQ10 decrease with age and in patients with heart failure, hypertension and diabetes. Coincidentally these are also risk factors for severe COVID-19 infection namely age, cardiovascular disease, lung disease, high blood pressure and cancer ${ }^{22}$

In fact, low plasma CoQ10 levels have been associated with acute influenza, viral papilloma infections and sepsis $^{23,24}$. An animal model of malaria showed CoQ10 treatment decreased inflammatory precursors, NFkB phosphorylation, and malondialdehyde and 8-hydroxy-2deoxyguanosine levels and restored cellular glutathione ${ }^{25}$. In addition, reduced levels of inflammation were observed in animal models of induced rheumatoid arthritis and acute pancreatitis after CoQ10 therapy ${ }^{26}$. Human clinical research has also verified the protective effects of CoQ10 supplementation prior to and after cardiovascular surgery in humans where it significantly supported patient outcomes and recovery post heart surgery ${ }^{27}$. Excessive levels of ROS found in critically ill COVID-19 patients upregulates cellular stress indicating that infected cells are struggling to cope with the increased metabolic demands during infection ${ }^{28,29}$. Essentially continued cellular oxidative stress from excessive SARS-CoV-2 viral loads has been linked with a high risk of patient death ${ }^{30}$. Also, a study which looked at CoQ10 depletion in acute influenza infections found that there was a correlation between low levels of CoQ10 and positive infection status as well as high expression of serum inflammatory cytokines ${ }^{31}$. 


\section{Vitamin D and Viral Infections}

Severe vitamin D deficiency has been found to be a predictor of negative outcomes in COVID-19 patients with respiratory failure; correlating with a $50 \%$ chance of poor prognosis and the need for assisted ventilation ${ }^{32,33}$. This risk is further amplified in the elderly with low vitamin D levels ${ }^{34}$. Crucially, vitamin D can directly decrease SARS-CoV-2 virulence by decreasing levels of dipeptidyl peptidase (DPP-4) / CD26 which is required to connect with the structural S1 domain of SARS-CoV-2 ${ }^{35,36}$. Vitamin D can also maintain respiratory homeostasis by supporting gap and cellular junctions, decreasing inflammatory cytokines but upregulating antiviral INF $\gamma$. Therefore, vitamin D could potentially divert abnormal cell responses in SARSCoV-2 mediated inflammation, cytokine storms and sepsis. Studies have also shown that chronic vitamin D deficiency can activate the renin angiotensin system (RAC) which promotes lung fibrosis and cardiovascular dysfunction ${ }^{37}$. Notably both of these conditions are known risk factors for severe COVID-19. Also, Vitamin D can increase ACE2 but reduce ACE production which is known to reduce inflammatory lung damage ${ }^{38}$. It is possible that Vitamin D could protect against COVID-19 symptoms since it is able to increase lung ACE2 expression which would stop renin synthesis. This in turn can decrease pro-inflammatory Angiotensin II action and so could potentially reduce ARDS risk as well as myocarditis and cardiac dysfunction ${ }^{39,40}$. In fact research has shown that each $4 \mathrm{ng} / \mathrm{ml}$ increase in 25 $(\mathrm{OH})$ D levels correlated with a 7\% reduction in respiratory infection risk ${ }^{41}$. A study from India by Rastogi et al 2020 was the first to report on the use of high dose Vitamin D supplementation and a higher incidence of recovery in COVID-19. After 7 days of treatment patients, who received vitamin D tested negative for SARS-CoV-2 RNA quicker than those with vitamin D deficiency. To note this small cohort of patients all had mild COVID-19 symptoms and no comorbidities $^{42}$.

\section{Mitochondrial Damage in Monocytes from COVID-19 Patients}

Interestingly, a study which analysed the blood monocytes derived from COVID-19 pneumonia patients in Italy found that the infected monocytes displayed alterations in mitochondrial functions and disrupted bioenergetics. The monocytes showed diminished aerobic glycolysis capability, reduced respiratory capacity, decreased proton leak and oxidative burst. The COVID-19 infected monocytes were able to produce cytokines which were skewed to a pro-inflammatory profile (TNF $\alpha$ ) rather than an INF $\gamma$ profile which stimulates an antiviral phenotype and is protective against viruses. Further analysis of monocyte subsets revealed that the COVID-19 monocytes were mostly immature, pro-inflammatory subsets with enhanced expression of inhibitory checkpoints. COVID-19 patient plasma also contained high levels of possibly monocyte derived cytokines and chemokines including IL-18, GM-CSF, CCL-2, CXCL10 and osteopontin which would inevitably contribute to chronic inflammation ${ }^{43}$. Cellular mitochondrial dysfunction and decreased ATP concentration has been previously correlated with multi organ failure, tissue hypoxia and sepsis $^{44,45}$. Figure 1 shows the hypothetical impact of SARSCoV-2 infection on immune mononuclear cell function. The bioenergetic failure of monocytes is extremely significant in COVID-19 pathogenesis as they are crucial immune cells involved in antigen presentation, phagocytosis and hence infection clearance. Longer illness and poor patient outcomes could occur if these cells are not able to function. Also, immune cells which are damaged could increase susceptibility of COVID-19 patients to secondary infections in the future. Recently this has been observed in India where thousands of recovered COVID-19 patients contracted mucormycosis or black fungus requiring urgent care and hospitilisation ${ }^{46}$. Although severe COVID-19 predominantly affects the lungs, the cytokine storms are also shown to affect the heart, endothelium and proximal blood vessels causing myocardial injury ${ }^{47}$. These patients could potentially benefit from the protective effects of CoQ10 which has been shown to be helpful during cellular recovery particularly after myocardial and pericardial tissue injury ${ }^{27}$.

\section{Conclusion and Future Perspectives}

A robust immune response to COVID-19 is essential; this requires healthy immune cells with functioning mitochondria and intact mucosal and adaptive immunity which can clear and resolve any inflammation caused by the virus. Age, ethnicity and virus tenacity are uncontrollable variables. However early interventions with CoQ10 and Vitamin D during SARS-CoV-2 infection could in theory support immune cell mitochondria bioenergetics, cell function, microbial phagocytosis, cell integrity therefore inflammation resolution and control. Further research should investigate COVID-19 patients with CoQ10 and vitamin $\mathrm{D}$ deficiency at presentation, with a view of profiling their clinical immune responses. The correlation between dual deficiencies in these two factors and serious or long COVID-19 is not known nor is the exact changes in CoQ10 and mitochondria function that accompany aging and differential genetics. A better understanding of viral spike protein interactions with ACE2 in CoQ10/ vitamin $\mathrm{D}$ deficient patients is also important as these binding receptors could form therapeutic targets on mucosal tissues, vascular endothelium and immune cells.

\section{Conflict of Interest}

The author confirms there is no conflict of interest. 


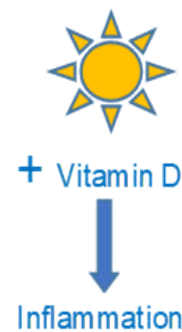

Resolution

\section{ARDS prevention} Sepsis prevention RAS Homeostasis

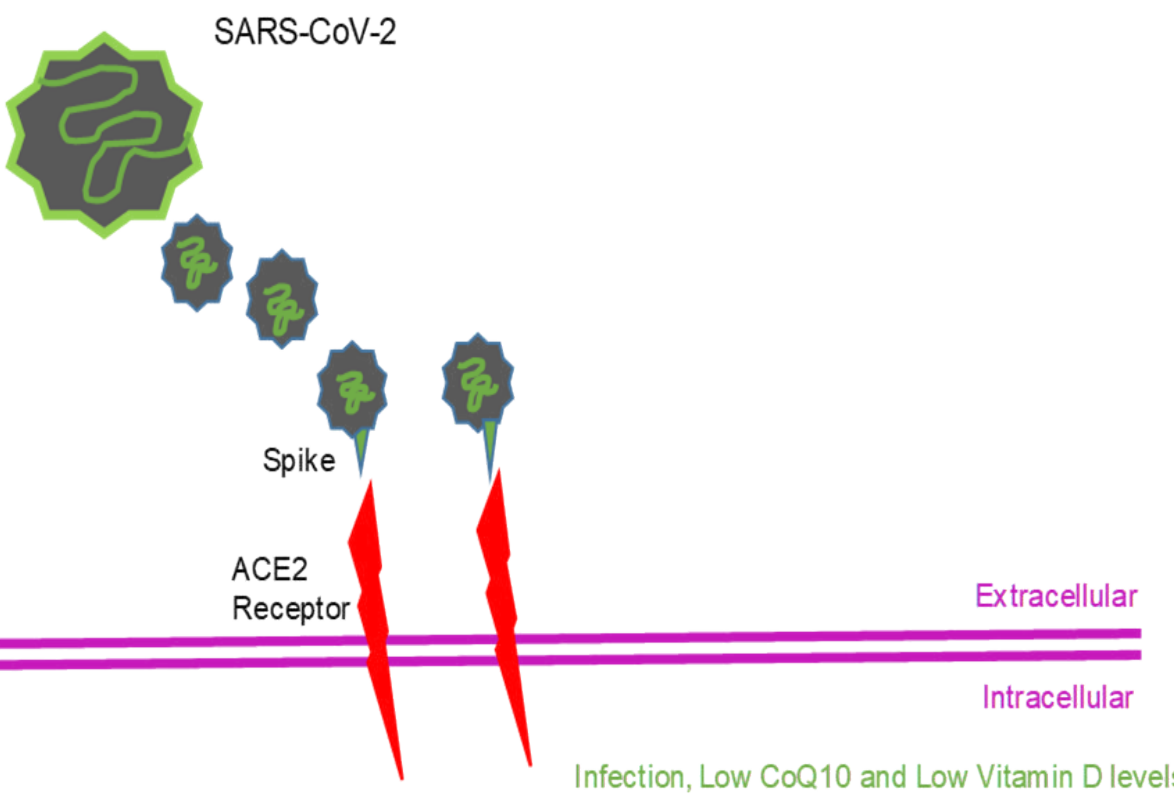

High viral load $=$ Increase in cell stress

\section{Decrease in cell microbe phagocytosis}

Decrease in respiratory burst Skewed cytokine release $>$ TNFa, $<$ INFY

Cytokine Storms, Inflammation, Sepsis

Increase in

Phagocytosis

Anti-inflammatory, Antioxidant Effects

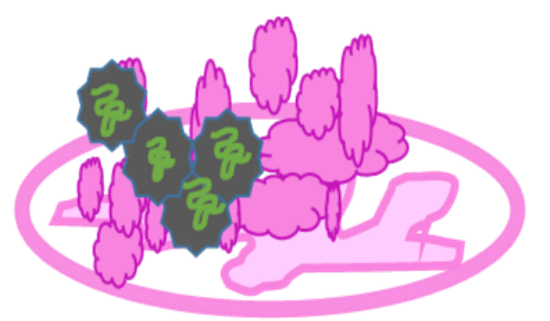

SARS-CoV-2 infection damages mitochondria causing DNA alteration \&

Figure 1. Hypothetical protective functions of Coenzyme Q10 and vitamin D in cells infected with SARS-CoV-2. As SARS-CoV-2 infects cells via the ACE2 receptor, it diminishes cellular energy by overtaking mitochondria and using adenine triose phosphate (ATP) for its purposes causing mitochondria stress and damage. In immune cells this leads to oxidative stress resulting in dysregulation of normal function such as key defences to microbes such as phagocytosis and oxidative burst. This would drive responses towards inflammation, cytokine storms and sepsis. Exogenous Vitamin D and coenzyme Q10 could ameliorate COVID-19 effects and support cellular recovery. Key: Angiotensin converting enzyme (ACE2), Coenzyme Q10 (CoQ10), Acute respiratory distress syndrome (ARDS), Renin angiotensin system (RAC).

\section{External Funding Received}

\section{None}

\section{References}

1. https://www.worldometers.info/coronavirus/ accessed $1^{\text {st }}$ July 2021

2. Ma X, Liang M, Ding M, et al. Extracorporeal Membrane Oxygenation (ECMO) in Critically Ill Patients with Coronavirus Disease 2019 (COVID-19) Pneumonia and Acute Respiratory Distress Syndrome (ARDS). Med Sci Monit. 2020 Aug 6; 26: e925364. doi: 10.12659/ MSM.925364.

3. Hu B, Huang S, Yin L. The cytokine storm and COVID-19. J Med Virol. 2021 Jan;93(1):250-256. doi: 10.1002/jmv.26232.

4. Costela-Ruiz VJ, Illescas-Montes R, Puerta-Puerta JM, et al. SARS-CoV-2 infection: The role of cytokines in COVID-19 disease. Cytokine Growth Factor Rev. 2020 Aug; 54: 62-75. doi: 10.1016/j.cytogfr.2020.06.001.

5. Coperchini F, Chiovato L, Croce L, et al. The cytokine storm in COVID-19: An overview of the involvement of the chemokine/ chemokine-receptor system. Cytokine Growth Factor Rev. 2020 Jun; 53: 25-32. doi: 10.1016/j.cytogfr.2020.05.003.

6. Schapkaitz E, De Jager T, Levy B. The characteristic peripheral blood morphological features of hospitalized patients infected with COVID-19. Int J Lab Hematol. 2021 Jun; 43(3): e130-e134. doi: 10.1111/ijlh.13417.

7. Khamis AH, Jaber M, Azar A, et al. Clinical and laboratory findings of COVID-19: A systematic review and meta-analysis. J Formos Med Assoc. 2020 Dec 15: S0929-6646(20)30603-3. doi: 10.1016/j. jfma.2020.12.003. 
8. Yang X, Yu Y, Xu J, et al. Clinical course and outcomes of critically ill patients with SARS-CoV-2 pneumonia in Wuhan, China: a singlecentered, retrospective, observational study. Lancet. 2020; 8: 475481. doi: 10.1016/S2213-2600(20)30079-5.

9. Gold MS, Sehayek D, Gabrielli S, et al. COVID-19 and comorbidities: a systematic review and meta-analysis. Postgrad Med. 2020 Nov; 132(8): 749-755. doi: 10.1080/00325481.2020.1786964.

10. Mackey K, Ayers CK, Kondo KK, et al. Racial and Ethnic Disparities in COVID-19-Related Infections, Hospitalizations, and Deaths: A Systematic Review. Ann Intern Med. 2021 Mar; 174(3): 362-373. doi 10.7326/M20-6306.

11. Kifer D, et al. Effects of Environmental Factors on Severity and Mortality of COVID-19. Front Med (Lausanne). 2021 Jan 20; 7 607786. doi: 10.3389/fmed.2020.607786.

12. Casas JP, Chua W, Loukogeorgakis S, et al. Effect of inhibitors of the renin-angiotensin system and other antihypertensive drugs on renal outcomes: systematic review and meta-analysis. Lancet. 2005 Dec 10 ; 366(9502): 2026-33. doi: 10.1016/S0140-6736(05)67814-2.

13. Trypsteen W, Van Cleemput J, Snippenberg WV, et al. On the whereabouts of SARS-CoV-2 in the human body: A systematic review. PLoS Pathog. 2020 Oct 30; 16(10): e1009037. doi: 10.1371/journal. ppat.1009037.

14. Li MY, Li L, Zhang Y, et al. Expression of the SARS-CoV-2 cell receptor gene ACE2 in a wide variety of human tissues. Infect Dis Poverty. 2020 Apr 28; 9(1): 45. doi: 10.1186/s40249-020-00662-x.

15. Gvozdjakova A, Klauco F, Kucharska J, et al. Is mitochondrial bioenergetics and coenzyme Q10 the target of a virus causing COVID-19? Bratisl Lek Listy. 2020; 121(11): 775-778. doi: 10.4149/ BLL_2020_126.

16. Seth RB, Sun L, Chen ZJ. Antiviral innate immunity pathways. Cell Res. 2006 Feb; 16(2): 141-7. doi: 10.1038/sj.cr.7310019.

17. Anand SK, Tikoo SK. Viruses as modulators of mitochondrial functions. Hindawi, Advances in Virology, Volume 2013; Article ID 738794, 17 pages; http: //dx.doi.org/10.1155/2013/738794.

18. Ohta A, Nishiyama Y. Mitochondria and viruses. Mitochondrion 2011; $11(1): 1-12$.

19. Ripoli M, D’Aprile A, Quarato G, et al. Hepatitis C-virus linked mitochondrial dysfunction promotes hypoxia-inducible factor 1 alphamediated glycolytic adaptation. J Virology 2010; 84 (1): 647-660.

20. Di Gennaro F, Pizzol D, Marotta C, et al. Coronavirus diseases (COVID-19) current status and future perspectives: A narrative review. Int J Environ Res Public Health 2020; 17, 2690. DOI: 10.3390/ ijerph17082690.

21. Garrido-Maraver J, Cordero MD, Oropesa-Avila M, et al. Clinical applications of coenzyme Q10. Front Biosci (Landmark Ed). 2014 Jan 1; 19: 619-33. doi: 10.2741/4231.

22. Gao YD, Ding M, Dong X, et al. Risk factors for severe and critically ill COVID-19 patients: A review. Allergy. 2021 Feb; 76(2): 428-455. doi: 10.1111/all.14657.

23. Guo J, Gao S, Liu Z, et al. Alpha-lipoic acid alleviates acute inflammation and promotes lipid mobilization during the inflammatory response in white adipose tissue of mice. Lipids. 2016; 51: 1145-1152. doi: 10.1007/s11745-016-4185-2.

24. Azmoonfar R, Amini P, Yahyapour R, et al. Mitigation of radiationinduced pneumonitis and lung fibrosis using alpha-lipoic acid and resveratrol. Antiinflamm Antiallergy Agent Med Chem. 2020; 19: 149157. doi: $10.2174 / 1871523018666190319144020$

25. Blanca AJ, Ruiz-Armenta MV, Zambrano S, et al. Leptin induces oxidative stress through activation of NADPH oxidase in renal tubular cells: antioxidant effect of L-carnitine. J Cell Biochem. 2016; 117: 2281-2288. doi: $10.1002 / j \mathrm{jb} .25526$.
26. Schmelzer C, Lindner I, Rimbach G, et al. Functions of coenzyme Q10 in inflammation and gene expression. BioFactors. 2008; 32: 179-183. doi: 10.1002/biof.5520320121.

27. Di Lorenzo A, Iannuzzo G, Parlato A, et al. Clinical Evidence for Q10 Coenzyme Supplementation in Heart Failure: From Energetics to Functional Improvement. J Clin Med. 2020 Apr 27; 9(5): 1266. doi: $10.3390 / \mathrm{jcm} 9051266$.

28. Laforge M, Elbim C, Frère $\mathrm{C}$, et al. Tissue damage from neutrophilinduced oxidative stress in COVID-19. Nat Rev Immunol. 2020;20(9):515-516. doi: 10.1038/s41577-020-0407-1.

29. de Las HN, Martín Giménez VM, Ferder L, et al. Implications of oxidative stress and potential role of mitochondrial dysfunction in COVID-19: therapeutic effects of Vitamin D. Antioxidants 2020; 9: 897. doi: $10.3390 /$ antiox9090897.

30. Varga Z, Flammer AJ, Steiger P, et al. Endothelial cell infection and endotheliitis in COVID-19. Lancet. 2020;395(10234):1417-1418. doi: 10.1016/S0140-6736(20)30937-5.

31. Chase M, Cocchi MN, Liu X, et al. Coenzyme Q10 in acute influenza. Influenza Other Respir Viruses. 2019 Jan;13(1):64-70. doi: 10.1111/ irv.12608.

32. Carpagnano GE, Di Lecce V, Quaranta VN, et al. Vitamin D deficiency as a predictor of poor prognosis in patients with acute respiratory failure due to COVID-19. J Endocrinol Invest. 2021 Apr; 44(4): 765771. doi: $10.1007 / \mathrm{s} 40618-020-01370-\mathrm{x}$.

33. Arvinte C, Singh M, Marik PE. Serum Levels of Vitamin C and Vitamin D in a Cohort of Critically Ill COVID-19 Patients of a North American Community Hospital Intensive Care Unit in May 2020: A Pilot Study. Med Drug Discov. 2020; 8: 100064. doi: 10.1016/j. medidd.2020.100064.

34. Baktash V, Hosack T, Patel N, et al. Vitamin D status and outcomes for hospitalised older patients with COVID-19. Postgrad Med J. 2021 Jul;97(1149):442-447. doi: 10.1136/ postgradmedj-2020-138712.

35. Vankadari N, Wilce J.A. Emerging WuHan (COVID-19) coronavirus: glycan shield and structure prediction of spike glycoprotein and its interaction with human CD26. Emerg Microb Infect. 2020; 9: 601-604.

36. Komolmit P, Charoensuk K, Thanapirom K, et al. Correction of vitamin D deficiency facilitated suppression of IP-10 and DPP IV levels in patients with chronic hepatitis C: a randomised double-blinded, placebo-control trial. PLOS ONE. 2017; 12

37. Shi Y, Liu T, Yao L, et al. Chronic vitamin D deficiency induces lung fibrosis through activation of the renin-angiotensin system. Sci Rep. 2017; 7: 1-10.

38. Kumar R, Rathi H, Haq A, et al. Putative roles of vitamin D in modulating immune response and immunopathology associated with COVID-19. Virus Res. 2021 Jan 15; 292: 198235. doi: 10.1016/j. virusres.2020.198235.

39. Andersen LB, Przybyl L, Haase N, et al. Vitamin D depletion aggravates hypertension and target-organ damage. J Am Heart Assoc. 2015 Jan 28; 4(2): e001417. doi: 10.1161/JAHA.114.001417.

40. Rastogi A, Bhansali A, Khare N, et al. Short term, high-dose vitamin D supplementation for COVID-19 disease: a randomised, placebocontrolled, study (SHADE study). Postgraduate Medical Journal. 2020. doi: $10.1136 /$ postgradmedj-2020-139065

41. Orkaby AR, Djousse L, Manson JE. Vitamin D supplements and prevention of cardiovascular disease. Curr Opin Cardiol. 2019 Nov; 34(6): 700-705. doi: 10.1097/HCO.0000000000000675.

42. Berry DJ, Hesketh K, Power C, et al. Vitamin D status has a linear association with seasonal infections and lung function in British adults. Br J Nutr. 2011 Nov; 106(9): 1433-40. doi: 10.1017/ S0007114511001991. 
43. Gibellini L, De Biasi S, Paolini A, et al. Altered bioenergetics and mitochondrial dysfunction of monocytes in patients with COVID-19 pneumonia. EMBO Mol Med. 2020 Dec 7; 12(12): e13001. doi: 10.15252/emmm.202013001.

44. McBride MA, Owen AM, Stothers CL, et al. The Metabolic Basis of Immune Dysfunction Following Sepsis and Trauma. Front Immunol. 2020 May 29; 11: 1043. doi: 10.3389/fimmu.2020.01043.

45. Brealey D, Brand M, Hargreaves I, et al. Association between mitochondrial dysfunction and severity and outcome of septic shock. Lancet. 2002 Jul 20; 360(9328): 219-23. doi: 10.1016/S01406736(02)09459-X.

46. Gandra S, Ram S, Levitz SM. The "Black Fungus" in India: The Emerging Syndemic of COVID-19-Associated Mucormycosis. Ann Intern Med. 2021 Jun 8. doi: 10.7326/M21-2354.

47. Ruzzenenti G, Maloberti A, Giani V, et al. Covid-19 Niguarda Working Group. Covid and Cardiovascular Diseases: Direct and Indirect Damages and Future Perspective. High Blood Press Cardiovasc Prev. 2021 Jun 26:1-7. doi: 10.1007/s40292-021-00464-8. 